University of Nebraska - Lincoln

DigitalCommons@University of Nebraska - Lincoln

1984

\title{
An Analysis of the Use of Rademacher-Walsh Spectrum in Compact Testing
}

Ten-Chuan Hsiao

California State University, Fullerton

Sharad C. Seth

University of Nebraska-Lincoln, seth@cse.unl.edu

Hsiao, Ten-Chuan and Seth, Sharad C., "An Analysis of the Use of Rademacher-Walsh Spectrum in Compact Testing" (1984). CSE Journal Articles. 36.

https://digitalcommons.unl.edu/csearticles/36

This Article is brought to you for free and open access by the Computer Science and Engineering, Department of at DigitalCommons@University of Nebraska - Lincoln. It has been accepted for inclusion in CSE Journal Articles by an authorized administrator of DigitalCommons@University of Nebraska - Lincoln. 
The performance of a random compact testing scheme can be characterized by two probabilities: the probability of rejecting a good unit and the probability of accepting a bad unit - the Type I and Type II errors, respectively, in statistics. In [7] Losq evaluates these measures for a random compact testing scheme in which the frequency of ones at the output is used as the signature. In Losq's scheme a random vector generator is used which produces a one at each of its outputs with a probability that can be varied to "fine-tune" the tester to the UUT. Thus, in [6]-[8] the signature is independent of the UUT but the random vector generator is dependent on it and chosen to minimize the two types of errors.

In contrast, the new random compact scheme analyzed in this correspondence has just the opposite characteristics: the random vector generator is independent of the UUT, producing a one at every output with the probability 0.5 ; the signature, on the other hand, is a coefficient chosen from the Rademacher-Walsh (RW) spectrum of the combinational function implemented by the UUT. The analysis suggests the choice of an RW coefficient with the largest possible magnitude so as to minimize the two types of errors. Finding an RW coefficient with the largest magnitude is NPcomplete (as is the problem of determining optimal input probability in earlier schemes). However, it may be possible to determine the same correctly with a high degree of confidence using less timeconsuming sampling techniques. A potential advantage of the proposed scheme is the ability of testing functionally different units using a tester with a programmable coefficient-selection logic.

\section{THE RADEMACHER-WALSH SPECTRA}

\section{An Analysis of the Use of Rademacher-Walsh Spectrum in Compact Testing}

\author{
TEN-CHUAN HSIAO AND SHARAD C. SETH
}

\begin{abstract}
Earlier approaches to random compact testing use a random pattern generator which depends on the combinational function under test and a circuit signature which remains the same independent of the circuit. In this correspondence we analyze the performance of a new scheme in which the pattern generator is simple and independent of the function being tested but the circuit signature is chosen to be a coefficient from the Rademacher-Walsh (RW) spectrum of the function under test. The analysis provides guidelines for choosing an RW coefficient, a test length, and an error tolerance so as to minimize the probabilities of rejecting a good unit or accepting a faulty one.
\end{abstract}

Index Terms - Error analysis, functional testing, Rademacher-Walsh coefficients, random compact testing.

\section{INTRODUCTION}

The standard way of testing digital circuits is to subject the unit under test (UUT) to a sequence of test vectors and compare its response against a reference response which is often just the output of a good unit to the same test stimuli. In compact testing schemes, however, the outputs of the UUT and the good unit are first compressed into a compact form before comparison. This simplifies the test procedure and reduces the cost of test application. Compact testing can be either deterministic [1]-[5] or random [6]-[8]. In this correspondence we analyze the performance of a new random compact testing scheme and provide guidelines for choosing its parameters in such a way so as to optimize its performance.

Manuscript received April 4, 1983; revised February 1, 1984.

T.- C. Hsiao was with the Department of Engineering Technology, Youngstown State University, Youngstown, $\mathrm{OH} 44503$. He is now with the Department of Electrical Engineering, California State University, Fullerton, CA 92634

S.C. Seth is with the Department of Computer Science, University of Nebraska, Lincoln, NE 68588.
The RW spectrum of a Boolean function $f(X)$ of $n$ variables consists of $2^{n}$ integer-valued coefficients characterizing $f(X)$ uniquely. The value of a coefficient ranges from $-2^{n}$ to $2^{n}$ and is always even. Each coefficient provides a measure of dependence of the function on a subset of input variables and will be denoted hereafter by $R(I)$ where $I$ is the set of indexes corresponding to the subset of input variables. The coefficients may be computed by using techniques similar to the fast Fourier transform [9] or by using the following formula [10], [11]:

$$
R(I)=2^{n}-2 \sum_{X}\left[f(X)+x\left(i_{1}\right)+x\left(i_{2}\right)+\cdots+x\left(i_{k}\right)\right]
$$

where

$$
\begin{aligned}
X= & \{x(1), x(2), \cdots, x(n)\}, \quad \text { the set of input variables, } \\
I= & \left\{i_{1}, i_{2}, \cdots, i_{k}\right\}, \quad \text { the set of variable indexes, and } \\
& \sum_{X} \text { denotes arithmetic summation ranging over the } 2^{n} \\
& \text { possible assignments to } X .
\end{aligned}
$$

It is customary to denote $R(I)$ as $R\left(i_{1} i_{2} \cdots i_{k}\right)$ and $R(I=$ null $)$ as $R(0)$. The spectrum of $f(X)$ is the vector

$$
S=[R(0), R(1), \cdots, R(n), R(12), \cdots, R(12 \cdots n)] .
$$

As an example, the spectrum of the "sum" function of a full adder is

$$
\begin{aligned}
S & =[R(0), R(1), R(2), R(3), R(12), R(13), R(23), R(123)] \\
& =[0,0,0,0,0,0,0,8] .
\end{aligned}
$$

This example points to an important fact for testing, namely that while each coefficient can be imagined as "encoding" partial information about the function, the amount of encoded information may vary widely. Indeed, for the "sum" function $R(123)$ by itself is enough to characterize the function uniquely. This observation is 


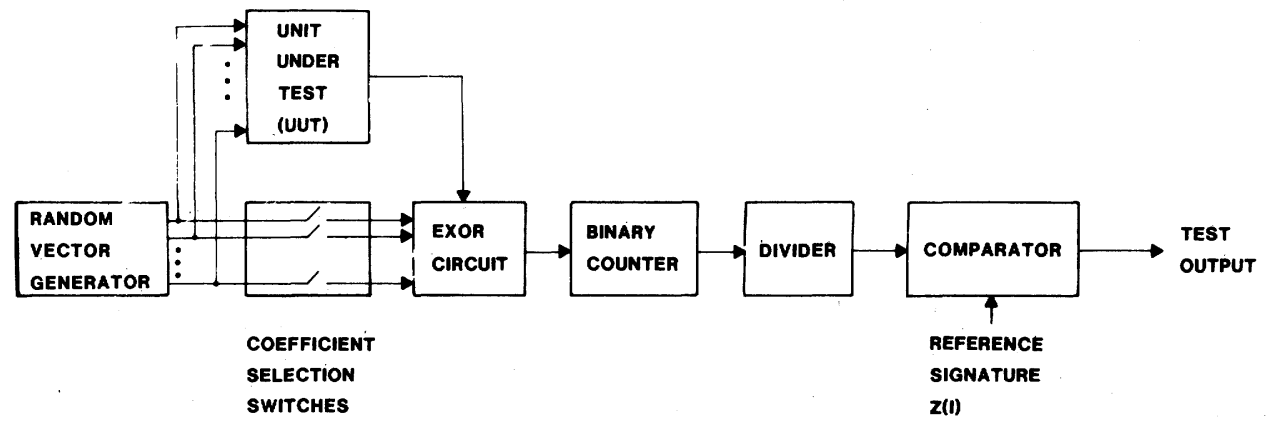

Fig. 1. A hardware diagram for implementing the RW compact testing scheme.

key to the use of a small number of RW coefficients as circuit signatures under very general fault assumptions. With equiprobable random test vectors the commonly used ones' count of a function is equivalent to an estimate of the coefficient $R(0)$ since, by (1), it is equal to $\left(2^{n}-R(0)\right) / 2[3]$.

\section{The Proposed TEST SCHEME}

The random compact tester employing an RW coefficient for signature is shown in Fig. 1. The random vector generator produces test vectors with equiprobable ones and zeros on each line. The output of the UUT and a selectable subset of the inputs are connected to an EXCLUSIVE OR (EXOR) circuit. The ones' count at the output of the EXOR circuit is accumulated for a test length of $T$ and normalized by dividing by $T$. The result is compared against the reference signature $Z(I)=0.5\left(1-R(I) / 2^{n}\right)$ where $R(I)$ is an RW coefficient with the largest magnitude. If the difference between the two signatures is less than a predetermined tolerance $\varepsilon$ the UUT is assumed to be fault free. Thus, the choice of the reference signature $Z(I)$, the test length $T$, and the tolerance $\varepsilon$ completely characterize this new testing scheme. We note parenthetically that the scheme of Fig. 1 can be generalized to test multiple outputs of a circuit concurrently by using a signature most appropriate for each output.

In the above discussion we assumed the knowledge of an RW coefficient with the largest magnitude for the given UUT and set the coefficient selection switches accordingly. However, by using the tester of Fig. 1 it is also possible to determine such a coefficient experimentally by varying the switch settings electronically and running randomly chosen sample tests for each setting. The larger the deviation of the divider output from 0.5 the larger will be the estimate for the corresponding coefficient. This may be particularly appropriate for testing a unit in the field without requiring knowledge of its complete functional description.

\section{AN ANALYSIS OF TEST PERFoRMANCE}

The Probability of Type I Error: Let $P_{1}$ denote the probability that a fault-free unit does not pass the test. Then

$$
P_{1}=1-\sum_{i=i_{1}}^{i_{2}}\left(\begin{array}{c}
T \\
i
\end{array}\right) Z(I)^{i}[1-Z(I)]^{T-i}
$$

where $i_{1}$ is the floor function of $T(Z(I)-\varepsilon)$ and $i_{2}$ is the ceiling function of $T(Z(I)+\varepsilon)$. From the Demoivre-Laplace limit theorem [12] $P_{1}$ can be approximated, for large values of $T$, as [9]

$$
\begin{gathered}
\frac{-(T \varepsilon+0.5)}{\sqrt{T Z(I)[1-Z(I)]}} \\
P_{1}=\frac{2}{\sqrt{2 \pi}} \int_{-\infty} e^{-0.5 t^{2}} d t
\end{gathered}
$$

which may be used to predict relative changes in the value of $P_{1}$ as the parameters $Z(I), T$, and $\varepsilon$ are varied. In particular, since the integrand is positive, a decrease in the upper limit of the integral will always result in a decrease in the value of $P_{1}$. Thus, $P_{1}$ decreases if a) the deviation of $Z(I)$ from 0.5 , i.e., $|Z(I)-0.5|$, increases, or b) the test length $T$ increases, or c) the tolerance $\varepsilon$ increases. Further, we notice that $|Z(I)-0.5|=\mid 0.5\left(1-R(I) / 2^{n}\right)-$ $0.5|=| R(I) / 2^{n+1} \mid$; therefore, assuming $T$ and $\varepsilon$ are fixed, $P_{1}$ can be reduced by choosing the largest magnitude coefficient in the RW spectrum of the function. By assuming $Z(I)=0.5$ [the value minimizing $P_{1}$ with respect to $\left.Z(I)\right]$ an upper bound can be obtained for the upper limit of the integral as

$$
\begin{aligned}
|-(T \varepsilon+0.5) / \sqrt{T Z(I)[1-Z(I)]}| \\
>|(T \varepsilon+0.5) / 0.5 \sqrt{T}| \simeq 2 \sqrt{T} \varepsilon
\end{aligned}
$$

which may be used in conjunction with the tables for the normal distribution (e.g., [12, pp. 176-177, Table 1]) to select the values of $T$ and $\varepsilon$. The above approximation also indicates that a tradeoff between $\varepsilon$ and $T$ is possible by varying $\varepsilon$ inversely as the square root of $T$.

The Probability of Type II Error: Let $P_{2}$ denote the probability of a faulty unit passing the test. $P_{2}$ cannot be estimated accurately without knowledge about the distribution of various kinds of faults. In [7] Losq provides persuasive arguments for the use of a functional fault model in which each of the $\left(2^{2^{n}}-1\right)$ faulty functions occur with equal probability; hence we choose to use this model in the following analysis.

Let $S=\left\{i / 2^{n} \mid i=0,1, \cdots, 2^{n}\right\}$ be the set of signatures possible at the output of a suspected faulty unit and let $Z(I)$ be the reference signature. Then

$$
P_{2}=\sum_{x \in S} p(x) \sum_{i=i_{1}}^{i_{2}}\left(\begin{array}{l}
T \\
i
\end{array}\right) x^{i}(1-x)^{T-i}
$$

where $i_{1}$ and $i_{2}$ are as defined above and $p(x)$ is the fraction of faulty units with signature $x$ given by

$$
p(x)=\left\{\begin{array}{l}
\left(\begin{array}{l}
2^{n} \\
2^{n} x
\end{array}\right) /\left(2^{2^{n}}-1\right), \quad x \neq Z(I) \\
{\left[\left(\begin{array}{l}
2^{n} \\
2^{n} x
\end{array}\right)-1\right] /\left(2^{2^{n}}-1\right), \quad x=Z(I) .}
\end{array}\right.
$$

As long as $\left(2^{n}{ }^{n} x\right)$ is much greater than one, $p(x)$ can be approximated as $\left(2^{n} x\right) /\left(2^{2^{n}}-1\right)$ which leads us to the final approximation we will use for $P_{2}$

$$
P_{2}=\sum_{x \in S}\left(\begin{array}{l}
2^{n} \\
2^{n} x
\end{array}\right) /\left(2^{2^{n}}-1\right) \sum_{i=i_{1}}^{i_{2}}\left(\begin{array}{l}
T \\
i
\end{array}\right) x^{i}(1-x)^{T-i} .
$$

The dependence of $P_{2}$ on parameters $Z(I), T$, and $\varepsilon$ is quite complex because of the double summation. However, an argument can be given (substantiated below by an example) that $P_{2}$ should decrease as $Z(I)$ deviates from 0.5 . First consider the factor $\left(2^{n}{ }^{n} x\right) /\left(2^{2^{n}}-1\right)$ in (4) which approximates $p(x)$. Obviously, this achieves its maximum value at $x=0.5$. Next, the term $\left(\begin{array}{l}T \\ i\end{array}\right) x^{i}(1-x)^{T-i}$ within the inner summation reaches its maximum when $x=i / T$; therefore, the inner summation itself should achieve 


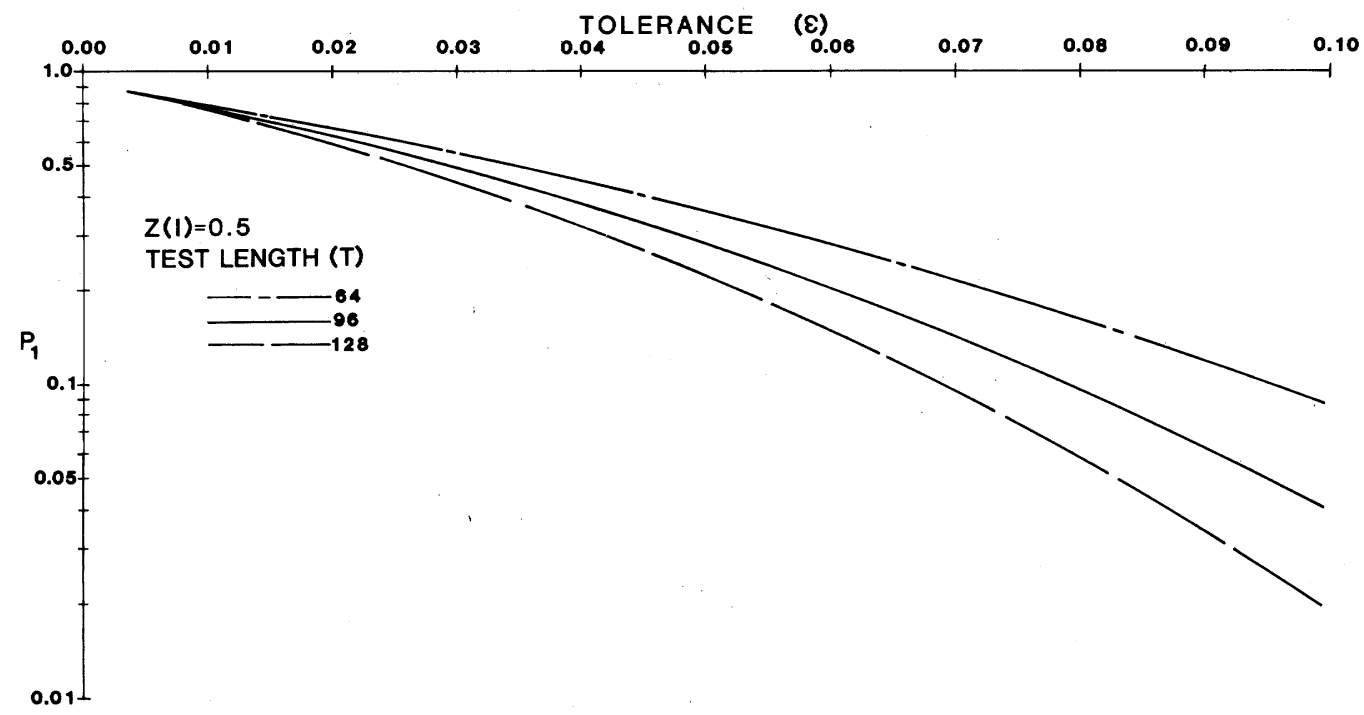

(a)

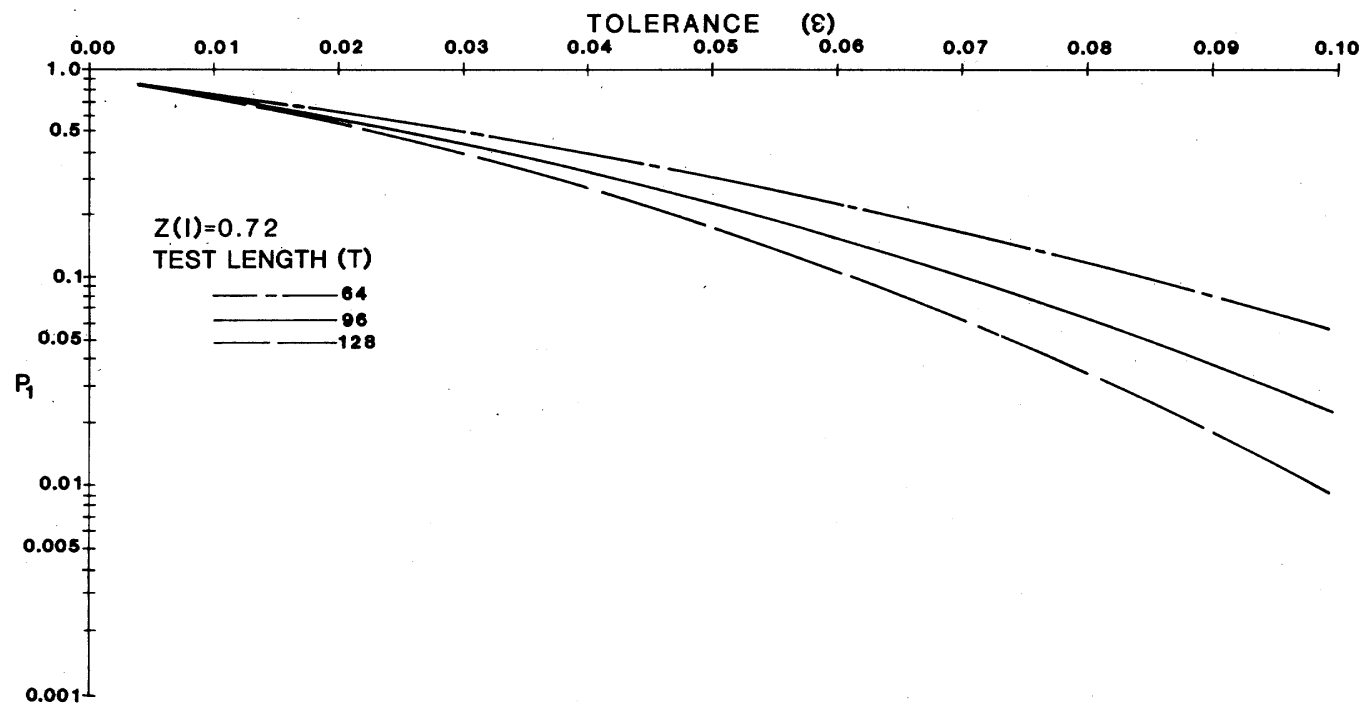

(b)

Fig. 2. The probability $P_{1}$ of rejecting a fault-free unit for the signature (a) $Z(I)=0.5$ and (b) $Z(I)=0.72$.

a large value when $x=Z(i)$ since $i$ ranges around $T Z(I)$. If $Z(I)$ is 0.5 then both factors in the outer summation have relatively high values causing $P_{2}$ to achieve a large value.

$P_{2}$ 's dependence on $\varepsilon$ is more straightforward. If the other two parameters are held fixed, $P_{2}$ will increase as $\varepsilon$ is increased because the inner summation in (4) is carried out over a larger number of terms. This also accords with our intuition: if the tolerance window is increased there is greater likelihood of a faulty unit registering a signature within the window.

Intuition would also indicate that $F_{2}$ should decrease as the test length $T$ is increased. Surprisingly, this is not always true, as the graph in Fig. 3 shows. The argument to explain this anomaly is quite long and can be found in [9].

An Example: Equations (2) and (4) can be used by the test engineer to determine whether random compact testing is adequate for a particular circuit, and if so which choices of the parameters are appropriate. For illustration, we consider a 7 -input circuit. In Fig. 2 $Z(I)$ and $T$ are used as parameters and the probability $P_{1}$ is shown as a function of the error tolerance $(\varepsilon)$ in a series of graphs. A similar series of graphs for $P_{2}$ are plotted in Fig. 3 using (4).

Suppose it is desired that the probability of a fault-free unit being rejected by a test be kept below 3 percent, independent of the circuit signature. This can be ensured by referring to the "worst-case" graph for $P_{1}$ with $Z(I)=0.5$ [Fig. 2(a)]. If a tolerance of 0.1 is used, the test length $T$ must be at least 128 . This conclusion could also have been arrived at by using the normal-distribution table along with (3). From Fig. 3(a), however, it is found that the probability of accepting a faulty unit is unacceptably high ( 40 percent) for $Z(I)=0.5$. Referring to the graph for $Z(I)=0.72$ we find that for the same test length and tolerance $P_{2}$ becomes less than 1 percent. $P_{1}$ also reduces to less than 1 percent for this choice of $Z(I)$.

We emphasize that the error probabilities $P_{1}$ and $P_{2}$ depend not on the absolute value of $Z(I)$ but on its deviation from 0.5 . Thus, in the above example, circuits with signatures $Z(I)>0.72$ would have the same error probabilities as circuits with $Z(I)<0.28$.

\section{CONCLUSION}

We considered a new scheme for random compact testing using a circuit-specific signature derived from a Rademacher-Walsh coefficient of the circuit function. Using standard statistical tools we analyzed the performance of this scheme in terms of the proba- 


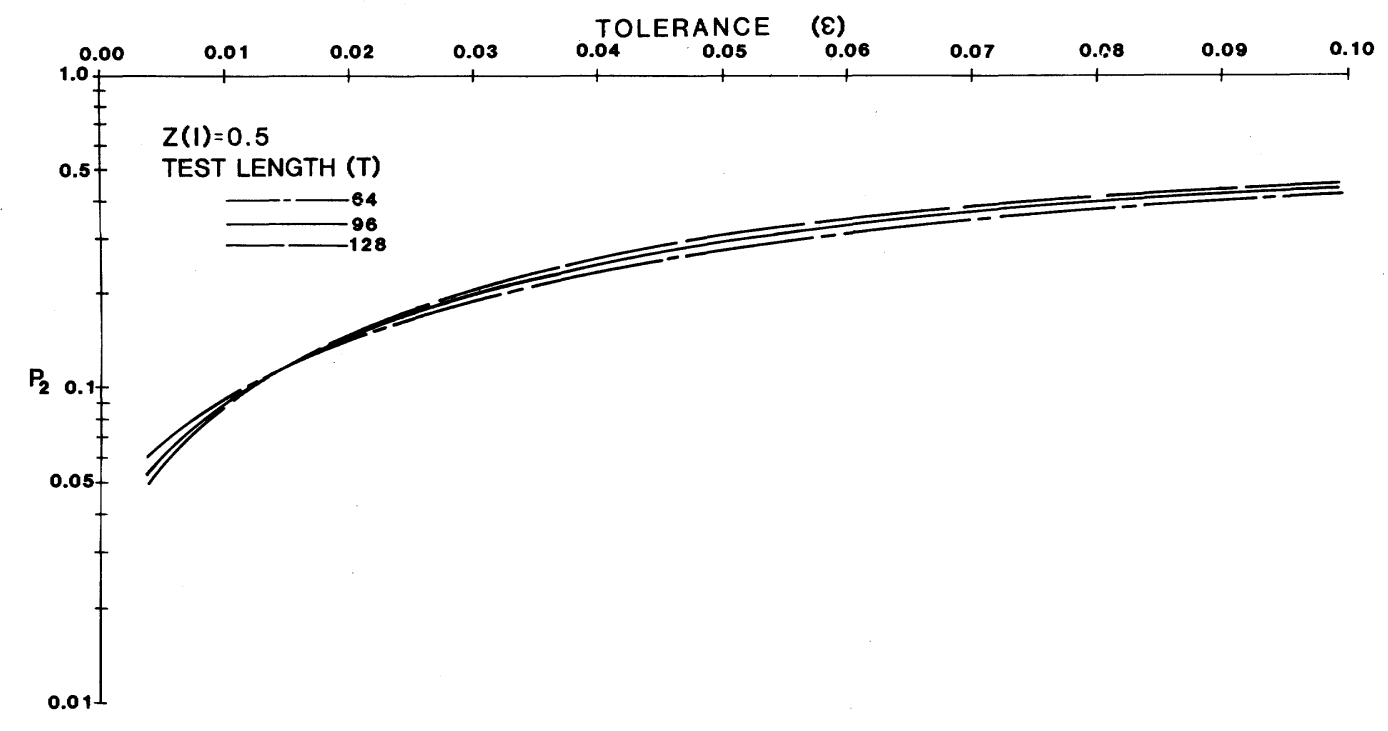

(a)

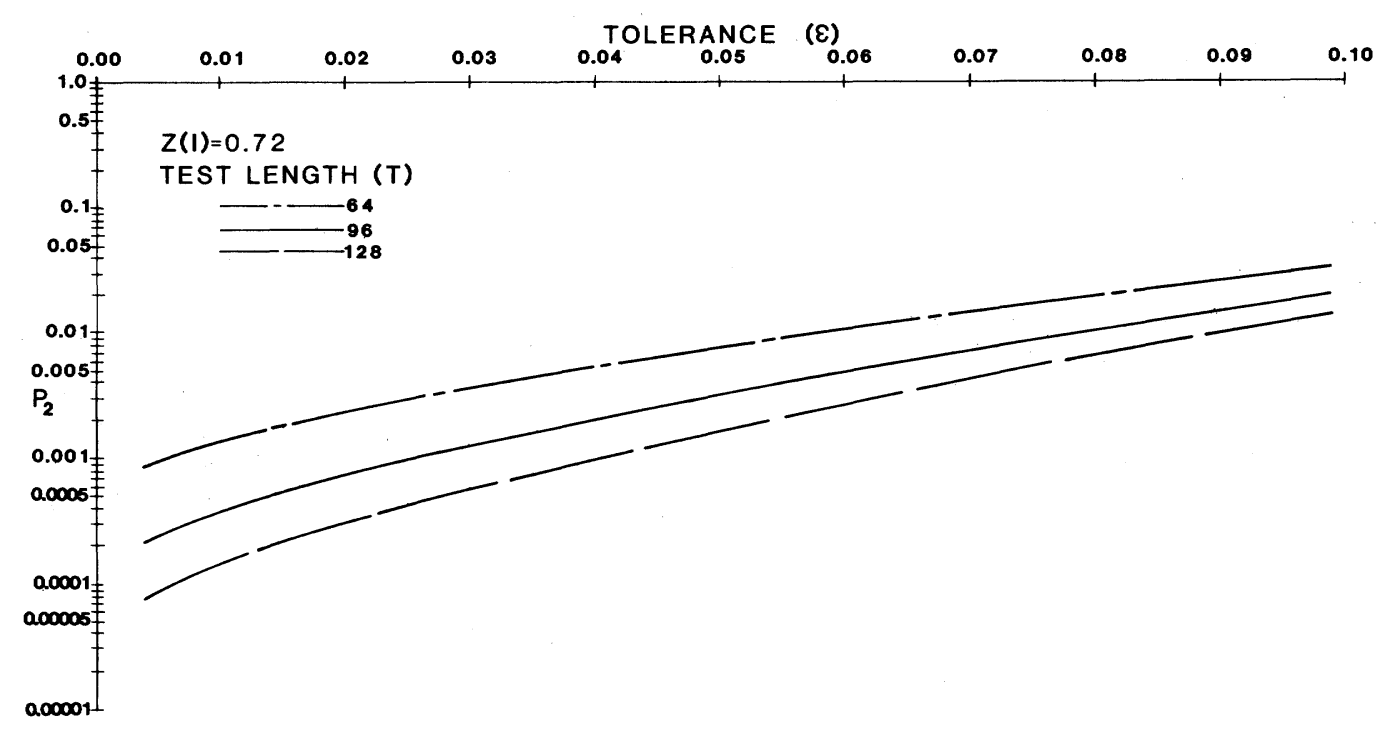

(b)

Fig. 3. The probability $P_{2}$ of accepting a faulty unit for the signature (a) $Z(I)=0.5$ and (b) $Z(I)=0.72$. The crossover of the curves in the first case is noteworthy.

bilities of rejecting a good unit and accepting a bad one. The results were shown to be useful in determining available tradeoffs between the circuit signature, the test length, and the test tolerance for a given level of performance.

One possible extension of the results reported here is to consider the effect of circuit modification on the RW spectrum of the resultant function. It appears that the measures, such as extra control inputs, to improve the testability of a circuit, also tend to move the signature values away from 0.5 (which was shown to be the worst signature value in the proposed test scheme). A rigorous proof of this hypothesis would be desirable.

\section{REFERENCES}

[1] J.P. Hayes, "Transition count testing of combinational logic circuits," IEEE Trans. Comput., vol. C-25, pp. 613-620, June 1976.

[2] J. Savir, "Syndrome-testable design of combinational circuits," IEEE Trans. Comput., vol. C-29, pp. 442-451, June 1980.

[3] A. K. Susskind, "Testing by verifying Walsh coefficients," in Proc. FTCS-11, Portland, ME, June 1981, pp. 206-208.
[4] A. Tzidon, I. Berger, and M. Yoeli, "A practical approach to fault detection in combinational networks," IEEE Trans. Comput., vol. C-27, pp. 968-971, Oct. 1978.

[5] J.C. Muzio and D. M. Miller, "Spectral techniques for fault detection," in Proc. FTCS-12, Santa Monica, CA, June 1982, pp. 297-302.

[6] J. Losq, "Referenceless random compact testing," in Proc. FTCS-6, Pittsburgh, PA, June 1976, pp. 108-113.

[7] _ , "Efficiency of random compact testing," IEEE Trans. Comput., vol. C-27, pp. 516-525, June 1978.

[8] K. P. Parker, "Compact testing: Testing with compressed data," in Proc. FTCS-6, Pittsburgh, PA, June 1976, pp. 93-98.

[9] T.C. Hsiao, "The use of Rademacher-Walsh spectrum for data compression in logic testing," Ph. D. dissertation, Univ. Nebraska, Lincoln, NE, Aug. 1982.

[10] T. C. Hsiao and S. C. Seth, "The use of Rademacher-Walsh spectrum in testing and design of digital circuits," in Proc. ICCC-82, New York, NY, Oct. 1982, pp. 202-205.

[11] S. L. Hurst, Logical Processing of Digital Signals. New York: Crane Russak, 1978.

[12] W. Feller, An Introduction to Probability Theory and Its Applications, Vol. 1. New York: Wiley, 1968. 